\title{
Effect of Prey Refuge and Harvesting on Dynamics of Eco-epidemiological Model with Holling Type III
}

\author{
Adin Lazuardy Firdiansyah ${ }^{1 *}$ \\ ${ }^{1}$ Department of Tadris Matematika, STAI Muhammadiyah Probolinggo, \\ Jl. Soekarno-Hatta No. 94B Kota Probolinggo 67219, Indonesia \\ *Corresponding Author. Email: adin.lazuardy@gmail.com
}

ABSTRACT

In this research, we formulate and analyze an eco-epidemiology model of the modified Leslie-Gower model with Holling type III by incorporating prey refuge and harvesting. In the model, we find at most six equilibrium where three equilibrium points are unstable and three equilibrium points are locally asymptotically stable. Furthermore, we find an interesting phenomenon, namely our model undergoes Hopf bifurcation at the interior equilibrium point by selecting refuge as the bifurcation parameter. Moreover, we also conclude that the stability of all populations occurs faster when the harvesting rate increases. In the end, several numerical solutions are presented to check the analytical results.

Keywords:

Eco-epidemiology model; Local stability; Hopf bifurcation

Citation Format:

A.L. Firdiansyah, "Effect of Prey Refuge and Harvesting on Dynamics of Eco-epidemiological Model with Holling Type III," Jambura J. Math., vol. 3, no. 1, pp. 16-25, 2021

\section{Introduction}

In the world of ecology, the interaction between predator and prey becomes an important issue in current research. One mathematical model described this issue is the Leslie-Gower model [1]. This model assumes that both populations grow logistically and the carrying capacity for predator depends on predator density. Currently, the predatorprey model has been modified to conform with actual conditions, such as the Allee effect [2][3], prey refuge [4][6], harvesting [4][7], etc.

In real life, the spread of infectious diseases may occur in the interaction between predator and prey. Therefore, this phenomenon has been considered by the mathematical model of epidemiology. As time goes by, ecology and epidemiology combine to form an eco-epidemiology model. Since then, several authors have investigated the eco-epidemiology model, as in [5][8].

In the eco-epidemiology model, especially the infected disease transmission in prey, the infectious disease can cause behavioral changes in prey that makes them easily caught by predators [9]. Therefore, the choice of the predator response function is an important thing to describe the amount of prey eaten by predator per unit time. Here, the predator response function used in the [5][8] is Holling type II form which represents that

e-ISSN: 2656-1344 @ 2021 A.L. Firdiansyah | Under the license CC BY-NC 4.0

Received: 18 September 2020 | Accepted: 15 October 2020 | Online: 2 January 2021 
predators occur maximum mortality when the prey density decrease. If the predator is more active hunting when prey density is getting large and less active when prey density decreases, then the Holling type III form can fit better [10]. The Leslie-Gower model and Holling type III has been investigated by several authors as in [6][11].

Another thing that is more relevant to the dynamic of the ecology model is the refuge of the prey population. Several authors as in [4][5][6] have investigated the dynamics of the ecology model including refuge in prey. They explain that prey refuge could influence the stability of the ecology model [4][5][6]. Moreover, it can prevent the extinction of the prey populations and give a factor which can be accessible to the predators [5]. Hence, the ecology model becomes more realistic.

We also know that the natural resource of ecology are predators and preys. Thus, both populations have commercial values and can be harvested in agriculture, fishery, forestry, and wildlife management. To investigate the effect of harvesting in the ecology model, several authors have been considered some harvesting functions, such as constant harvesting, proportionate harvesting, and nonlinear harvesting [4][7]. According to [9], the harvesting function has a stabilizing effect on the dynamics of the eco-epidemiology model.

Base on the above motivation, we formulate and analyze an eco-epidemiology model of the modified Leslie-Gower model as in [5] by changing predator functional response into Holling type III and including the effect of harvesting in the healthy preys. We also consider the effect of refuge in the infected prey. Thus, the model formulation analyzed here is a different model and more realistic than the previous literature. The main topic in our work is to observe the dynamics behavior and influence of harvesting in the model formulation.

\section{Methods}

In this part, we use several methods to investigate the dynamics of our model formulation. These methods are presented as follows:

1. Reviewing and studying the Leslie-Gower model from previous literature.

2. Modifying the Leslie-Gower model by including Holling type III and adding the refuging and harvesting on prey.

3. Identifying the existence of the equilibrium point of the model formulation.

4. Analyzing the dynamic stability and Hopf bifurcation of the model formulation.

5. Illustrating the numerical solution of the model formulation by using $4^{\text {th }}$-order Runge-Kutta schemes. This step is used to check the theoretical results.

6. Making conclusions from the theoretical results.

\section{Results and discussion}

In the construction model, populations are divided into three species. The first is susceptible to prey populations $S(t)$ that is likely to become infected. The second is infected prey populations $I(t)$ that has been infected by a certain disease and can spread disease. The third is predator populations $Y(t)$ that can only predate infected prey populations. An important note in the construction model is the infected population doesn't recover or become immune and disease in an infected population doesn't infect predators through food or other means. 


\subsection{Model formulation}

The assumptions used in the construction model is only susceptible prey populations experience harvesting, predators eat infected prey populations by following Holling type III function response, and incorporating the effect of refuge in the infected prey. Base on the assumptions given, the mathematical model can be presented by ordinary differential equations as follows:

$$
\begin{aligned}
& \dot{S}=a_{1} S-b S(S+I)-\beta S I-h S, \\
& \dot{I}=\beta S I-\frac{p_{1}(1-\sigma)^{2} I^{2} Y}{k+(1-\sigma)^{2} I^{2}}-d I, \\
& \dot{Y}=\left(a_{2}-\frac{p_{2} Y}{k+(1-\sigma) I}\right) Y .
\end{aligned}
$$

All parameters with the biological meaning can be summarized in the following table 1 . We assume that all parameters and initial conditions are positive values.

Table 1. Biological interpretation of system (1)

\begin{tabular}{cl}
\hline Parameters & \multicolumn{1}{c}{ Biological interpretations } \\
\hline$a_{1}$ & The intrinsic growth rate of susceptible prey \\
$a_{2}$ & The intrinsic growth rate of the predator \\
$k$ & The half-saturation constant \\
$b$ & The measure of the strength of the prey \\
$d$ & interference competition \\
$h$ & The death rate of infected prey \\
$\sigma$ & The harvesting rate \\
$p_{1}$ & The preditition rate \\
$p_{2}$ & The subtraction rate of the predator \\
$\beta$ & The infection rate \\
\hline
\end{tabular}

Source: [5][7]

\subsection{The existence of equilibrium points}

By setting the right-hand side equal zero, we obtain the possible equilibrium points. Thus, the equilibrium points of the system (1) are below:

1. The trivial equilibrium point is $E_{0}(0,0,0)$, which means that the extinction of both prey and predator. The point $E_{0}$ always exists.

2. The axial equilibrium points are $E_{1}\left(\frac{a_{1}-h}{b}, 0,0\right)$ and $E_{2}\left(0,0, \frac{k a_{2}}{p_{2}}\right)$. The point $E_{1}$ means that the extinction of infected prey and predator. This point exists if $a_{1}>h$. Meanwhile, the point $E_{2}$ means that the extinction of both preys. For point $E_{2}$, it always exists.

3. The planar equilibrium points are $E_{3}(\bar{S}, \bar{I}, 0)$ with $\bar{S}=\frac{d}{\beta} ; \bar{I}=\frac{\beta\left(a_{1}-h\right)-d b}{\beta(b+\beta)}$ and $E_{4}(\hat{S}, 0, \hat{Y})$ with $\hat{S}=\frac{a_{1}-h}{b} ; \hat{Y}=\frac{k a_{2}}{p_{2}}$. The point $E_{3}$ means that the extinction of 
predators. This point $E_{3}$ exists when $\beta a_{1}>\beta h+d b$. Meanwhile, the point $E_{4}$ exists if $a_{1}>h$. This point $E_{4}$ means that the extinction of infected prey.

4. The interior equilibrium point is $E^{*}\left(S^{*}, I^{*}, Y^{*}\right)$ where $S^{*}=\frac{a_{1}-b I^{*}-\beta I^{*}-h}{b}, Y^{*}=$ $\frac{a_{2}\left(k+(1-\sigma) I^{*}\right)}{p_{2}}$, and $I^{*}$ are the positive root of cubic equation $\vartheta\left(I^{*}\right)^{3}+\theta\left(I^{*}\right)^{2}+\mu I^{*}+\gamma=$ 0 with

$$
\begin{aligned}
& \vartheta=(1-\sigma)^{2} p_{2} \beta(b+\beta), \\
& \theta=(1-\sigma)^{2}\left[(1-\sigma) p_{1} b a_{2}+b d p_{2}+\beta p_{2} h-\beta p_{2} a_{1}\right], \\
& \mu=k\left[(1-\sigma)^{2} p_{1} b a_{2}+b \beta p_{2}+\beta^{2} p_{2}\right], \\
& \gamma=k p_{2}\left(b d+\beta h-\beta a_{1}\right) .
\end{aligned}
$$

The existence and explicit form is obtained by using Cardan's method as in [2].

\subsection{The stability analysis}

In this part, we observe the local stability of each equilibrium point. To check the dynamics around the equilibrium point, we determine eigenvalues of the Jacobi matrix at equilibrium point $E(S, I, Y)$. Here, we identify the Jacobi matrix as follows:

$$
J(E)=\left[\begin{array}{ccc}
u_{11} & u_{12} & 0 \\
u_{21} & u_{22} & u_{23} \\
0 & u_{32} & u_{33}
\end{array}\right],
$$

where

$$
\begin{aligned}
& u_{11}=a_{1}-b(S+I)-b S-\beta I-h, \\
& u_{12}=-S(b+\beta), u_{21}=\beta I, \\
& u_{22}=\beta S-\frac{2 k p_{1}(1-\sigma)^{2} I Y}{\left[k+(1-\sigma)^{2} I^{2}\right]^{2}}-d, \\
& u_{23}=-\frac{p_{1}(1-\sigma)^{2} I^{2}}{k+(1-\sigma)^{2} I^{2}}, \\
& u_{32}=\frac{p_{2}(1-\sigma) Y^{2}}{[k+(1-\sigma) I]^{2}}, \\
& u_{33}=-\frac{2 p_{2} Y}{k+(1-\sigma) I}+a_{2} .
\end{aligned}
$$

To analyze the stability of the equilibrium point $E_{0}$, we replace $E(S, I, Y)$ in equation (3) with $E_{0}$. Hence, the Jacobi matrix at $E_{0}$ becomes

$$
J\left(E_{0}\right)=\left[\begin{array}{ccc}
a_{1}-h & 0 & 0 \\
0 & -d & 0 \\
0 & 0 & a_{2}
\end{array}\right] .
$$

and eigenvalues of $J\left(E_{0}\right)$ are $\lambda_{1}=a_{1}-h ; \lambda_{2}=-d ; \lambda_{3}=a_{2}$. It is confirmed that $\lambda_{3}>0$, which means that the equilibrium point $E_{0}$ is unstable. Thus, we have the following theorem.

Theorem 1. The trivial equilibrium point $E_{0}$ is unstable.

Next, we shall investigate the axial equilibrium $E_{1}$ and $E_{2}$. First, to investigate the equilibrium point $E_{1}$, we substitute $E_{1}$ to equation (3). Hence, the Jacobi matrix at $E_{1}$ 


\section{Adin Lazuardy Firdiansyah}

becomes

$$
J\left(E_{1}\right)=\left[\begin{array}{ccc}
h-a_{1} & -a_{1}+h-\frac{\beta\left(a_{1}-h\right)}{b} & 0 \\
0 & \frac{\beta\left(a_{1}-h\right)}{b}-d & 0 \\
0 & 0 & a_{2}
\end{array}\right] .
$$

Thus, we have eigenvalues of the characteristic equation of $J\left(E_{1}\right)$, i.e. $\lambda_{1}=h-a_{1} ; \lambda_{2}=$ $\frac{\beta\left(a_{1}-h\right)}{b}-d ; \lambda_{3}=a_{2}$. It is confirmed that $\lambda_{3}>0$. Therefore, the equilibrium $E_{1}$ is unstable. Second, to identify the stability of $E_{2}$, we substitute $E_{2}$ to equation (3). Hence, the Jacobi matrix at $E_{2}$ becomes

$$
J\left(E_{2}\right)=\left[\begin{array}{ccc}
a_{1}-h & 0 & 0 \\
0 & -d & 0 \\
0 & \frac{a_{2}^{2}(1-\sigma)}{p_{2}} & -a_{2}
\end{array}\right] .
$$

Thus, we have eigenvalues of the characteristic equation of $J\left(E_{2}\right)$, namely $\lambda_{1}=a_{1}-$ $h ; \lambda_{2}=-d ; \lambda_{3}=-a_{2}$. It is easily confirmed that $\lambda_{1}<0$ when $a_{1}<h$. Therefore, the equilibrium $E_{2}$ is locally asymptotically stable if and only if $a_{1}<h$. Thus, we get the following theorem.

Theorem 2. The axial equilibrium $E_{1}$ is unstable and other axial equilibrium $E_{2}$ is locally asymptotically stable if $a_{1}<h$.

Next, we shall identify the stability of the planar equilibrium $E_{3}$ and $E_{4}$. First, we investigate the stability of the equilibrium point $E_{3}$. By substituting $E_{3}$ to equation (3), the Jacobi matrix at $E_{3}$ becomes

$$
J\left(E_{3}\right)=\left[\begin{array}{ccc}
-b \bar{S} & -\bar{S}(b+\beta) & 0 \\
\beta \bar{I} & 0 & -\frac{p_{1}(1-\sigma)^{2}(\bar{I})^{2}}{k+(1-\sigma)^{2}(\bar{I})^{2}} \\
0 & 0 & a_{2}
\end{array}\right]
$$

Therefore, eigenvalues of the characteristic equation of $J\left(E_{3}\right)$ is $\lambda_{1}=a_{2}$ and positive roots of a quadratic equation $\lambda^{2}+Q_{1} \lambda+Q_{2}=0$, where $Q_{1}=b \bar{S} ; Q_{2}=\beta(b+\beta) \bar{S} \bar{I}$. It is easily confirmed that one of the eigenvalues $\lambda_{1}>0$, which means that the equilibrium point $E_{3}$ is unstable. Second, we identify the stability of the equilibrium point $E_{4}$. By substituting $E_{4}$ to equation (3), the Jacobi matrix at $E_{4}$ becomes

$$
J\left(E_{4}\right)=\left[\begin{array}{ccc}
h-a_{1} & -a_{1}+h-\frac{\beta\left(a_{1}-h\right)}{b} & 0 \\
0 & \frac{\beta\left(a_{1}-h\right)}{b}-d & 0 \\
0 & \frac{a_{2}^{2}(1-\sigma)}{p_{2}} & -a_{2}
\end{array}\right] .
$$

Therefore, eigenvalues of the characteristic equation of $J\left(E_{4}\right)$ is $\lambda_{1}=h-a_{1} ; \lambda_{2}=$ 
$\frac{\beta\left(a_{1}-h\right)}{b}-d ; \lambda_{3}=-a_{2}$. It can be confirmed that $\lambda_{1}<0$ when $h<a_{1}$ and $\lambda_{2}<0$ when $\beta\left(a_{1}-h\right)<d b$. Thus, the equilibrium point $E_{4}$ is locally asymptotically stable when $h<$ $a_{1}$ and $\beta\left(a_{1}-h\right)<d b$. Furthermore, we obtain the following theorem.

Theorem 3. The planar equilibrium $E_{3}$ is unstable and other planar equilibrium $E_{4}$ is locally asymptotically stable if $h<a_{1}$ and $\beta\left(a_{1}-h\right)<d b$.

The last point is an interior equilibrium point $E^{*}$. To identify the stability of $E^{*}$, we have to substitute $E^{*}$ to equation (3). Hence, the Jacobi matrix at $E^{*}$ becomes

$$
J\left(E^{*}\right)=\left[\begin{array}{ccc}
v_{11} & v_{12} & 0 \\
v_{21} & v_{22} & v_{23} \\
0 & v_{32} & v_{33}
\end{array}\right],
$$

where

$$
\begin{aligned}
& v_{11}=-b S^{*}, v_{12}=-S^{*}(b+\beta), v_{21}=\beta I^{*}, \\
& v_{22}=\frac{p_{1}(1-\sigma)^{4}\left(I^{*}\right)^{3} Y^{*}}{\left[k+(1-\sigma)^{2}\left(I^{*}\right)^{2}\right]^{2}}-\frac{k p_{1}(1-\sigma)^{2} I^{*} Y^{*}}{\left[k+(1-\sigma)^{2}\left(I^{*}\right)^{2}\right]^{2}}, \\
& v_{23}=-\frac{p_{1}(1-\sigma)^{2}\left(I^{*}\right)^{2}}{k+(1-\sigma)^{2}\left(I^{*}\right)^{2}}, \\
& v_{32}=\frac{p_{2}(1-\sigma)\left(Y^{*}\right)^{2}}{\left[k+(1-\sigma) I^{*}\right]^{2}}, v_{33}=-\frac{p_{2} Y^{*}}{k+(1-\sigma) I^{*}} .
\end{aligned}
$$

Thus, the characteristic equation of $E^{*}$ is

$$
\lambda^{3}+\varphi_{1} \lambda^{2}+\varphi_{2} \lambda+\varphi_{3}=0
$$

where

$$
\begin{aligned}
& \varphi_{1}=-\left(v_{11}+v_{22}+v_{33}\right) \\
& \varphi_{2}=v_{11} v_{22}+v_{11} v_{33}+v_{22} v_{33}-v_{12} v_{21}-v_{23} v_{32} \\
& \varphi_{3}=v_{11} v_{23} v_{32}+v_{21} v_{12} v_{33}-v_{11} v_{22} v_{33} .
\end{aligned}
$$

By using the Routh-Hurwitz criterion, all eigenvalues of equation (12) have negative real roots if and only if $\varphi_{1}>0 ; \varphi_{3}>0 ; \varphi_{1} \varphi_{2}>\varphi_{3}$. Therefore, it proves that the equilibrium point $E^{*}$ is locally asymptotically stable. Thus, we get a theorem as follows.

Theorem 4. The equilibrium point $E^{*}$ is locally asymptotically stable if $E^{*}$ satisfies the condition $\varphi_{1}>0 ; \varphi_{3}>0 ; \varphi_{1} \varphi_{2}>\varphi_{3}$.

Finally, we shall identify a condition where the system (1) enters Hopf bifurcation. Hopf bifurcation guarantees the change of stability which means that our system has fluctuating populations. Here, we take $\sigma$ as bifurcation parameters that can influence dynamic behavior. The next theorem guarantees that there is a limit cycle over the interior point.

Theorem 5. The system (1) penetrates Hopf bifurcation over $E^{*}$ when $\sigma$ crosses a critical value $\sigma=\sigma_{c}$.

Proof. We start by giving a necessary condition for Hopf bifurcation at $\sigma=\sigma_{c}$ as fellows: 
a. $\varphi_{1}>0, \varphi_{3}>0$, and $\varphi_{1}\left(\sigma_{c}\right) \varphi_{2}\left(\sigma_{c}\right)-\varphi_{3}\left(\sigma_{c}\right)=0$.

b. $\left.\frac{d}{d \sigma}(\operatorname{Re}(\lambda(\sigma)))\right|_{\sigma=\sigma_{c}} \neq 0$.

For $\sigma=\sigma_{c}$, if we apply conditions $\varphi_{1}\left(\sigma_{c}\right) \varphi_{2}\left(\sigma_{c}\right)-\varphi_{3}\left(\sigma_{c}\right)=0$ then equation (12) becomes

$$
\left(\lambda^{2}+\varphi_{2}\right)\left(\lambda+\varphi_{1}\right)=0
$$

Hence, the solution of equation (14) is the strictly negative eigenvalue $\lambda_{1}=-\varphi_{1}$ and a pair of imaginary eigenvalues $\lambda_{2,3}= \pm i \sqrt{\varphi_{2}}$. For $\sigma$ at near of $\sigma_{c}$, the solution of equation (14) has the form $\lambda_{1}=-\varphi_{1}(\sigma) ; \lambda_{2}=e_{1}(\sigma)-i e_{2}(\sigma) ; \lambda_{3}=e_{1}(\sigma)+i e_{2}(\sigma)$ where $e_{1}(\sigma), e_{2}(\sigma)$ are real. Next, we shall prove that the transversality condition isn't equal to zero, i.e.

$$
\left.\frac{d}{d \sigma}\left(\operatorname{Re}\left(\lambda_{j}(\sigma)\right)\right)\right|_{\sigma=\sigma_{c}} \neq 0, j=2,3
$$

Now, by substituting $\lambda_{3}=e_{1}(\sigma)+i e_{2}(\sigma)$ into equation (14) and calculating derivative to $\sigma$, we obtain

$$
\begin{aligned}
& B_{1} \dot{e_{1}}-B_{2} \dot{e_{2}}+B_{3}=0 \\
& B_{2} \dot{e_{1}}+B_{1} \dot{e_{2}}+B_{4}=0
\end{aligned}
$$

where the form $B_{1}=3\left(e_{1}^{2}-e_{2}^{2}\right)+\varphi_{2}+2 e_{1} \varphi_{1} ; B_{2}=6 e_{1} e_{2}+2 e_{2} \varphi_{1} ; B_{3}=\dot{\varphi}_{1}\left(e_{1}^{2}-e_{2}^{2}\right)+$ $e_{1} \dot{\varphi_{2}}+\dot{\varphi_{3}} ; B_{4}=2 e_{1} e_{2} \dot{\varphi_{1}}+e_{2} \dot{\varphi_{2}}$. By solving equation (16), we get

$$
\dot{e_{1}}=-\frac{B_{2} B_{4}+B_{1} B_{3}}{B_{1}^{2}+B_{2}^{2}}
$$

Since $B_{2} B_{4}+B_{1} B_{3} \neq 0$ and $\dot{\varphi_{1}} \neq 0$, we obtain

$$
\left.\frac{d}{d \sigma}\left(\operatorname{Re}\left(\lambda_{j}(\sigma)\right)\right)\right|_{\sigma=\sigma_{c}}=-\left.\frac{B_{2} B_{4}+B_{1} B_{3}}{B_{1}^{2}+B_{2}^{2}}\right|_{\sigma=\sigma_{c}} \neq 0, j=2,3
$$

and $\lambda_{1}\left(\sigma_{c}\right)=-\varphi_{1}\left(\sigma_{c}\right) \neq 0$. Hence, it proves that the transversality condition is satisfied. It means that the equilibrium point $E^{*}$ undergoes Hopf bifurcation at $\sigma=\sigma_{c}$.

\subsection{Numerical solutions}

In this part, we shall illustrate our analytical results as in the previous part. By using $4^{\text {th }}$ order Runge-Kutta schemes as a numerical method with $\Delta t=0.01$, we perform some numerical solutions to demonstrate the dynamics of the system (1). For simulation purposes, we use hypothetical parameter values in the absence of field data. We select some parameter values as follows: 


$$
\begin{aligned}
a_{1}=5.5, a_{2}=0.2, k=0.2, b & =0.3, p_{1}=1.4, p_{2}=0.4, d=0.6, \beta=0.09, \\
\sigma & =0.42, h=0.7 .
\end{aligned}
$$

By using the parameter values as in equation (19), we show that system (1) has six existence of equilibrium points, namely $E_{0}(0,0,0), E_{1}(16,0,0), E_{2}(0,0,0.1)$, $E_{3}(6.67,0,7.18,0), E_{4}(16,0,0.1)$ and $E^{*}(11.393,3.543,1.128)$ and the condition of theorem 4 is satisfied. Hence, the equilibrium point $E^{*}$ is locally asymptotically stable (see figure $1(\mathrm{~b})$ ) and all trajectories are convergent to $E^{*}$ (see figure $1(\mathrm{a})$ ).

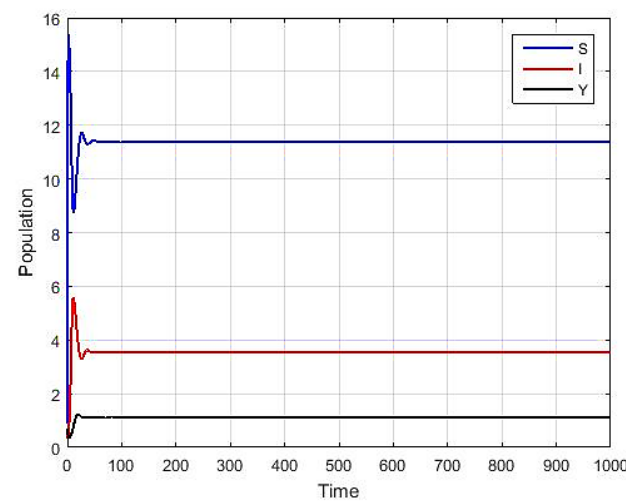

(a)

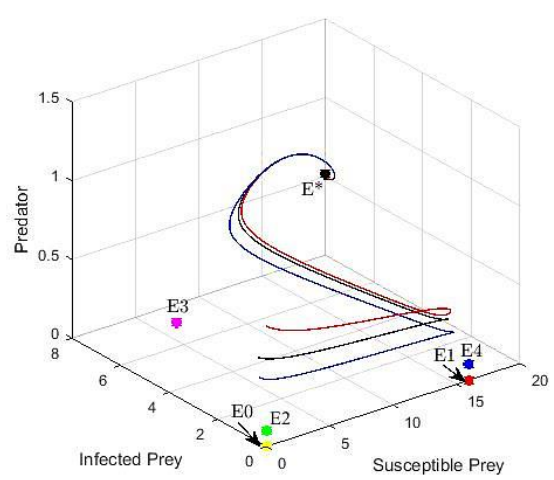

(b)

Figure 1. Numerical solution for $E^{*}$ : (a) time plot, (b) the phase portrait

To identify the system (1) undergoes Hopf bifurcation, we take $\sigma$ for the bifurcation parameter. If we select $\sigma=\sigma_{c}=0.26$, then the periodic solution enters Hopf bifurcation when $\sigma=\sigma_{c}=0.26$. Thus, the condition of theorem 5 is satisfied. Next, if we select $\sigma=$ $0.21<\sigma_{c}=0.26$, then the interior equilibrium point is unstable and there is a limit cycle around $E^{*}$ (see figure 2(a)). Meanwhile, when $\sigma=0.3>\sigma_{c}=0.26$, the interior equilibrium point $E^{*}$ is stable (see figure $2(b)$ ).

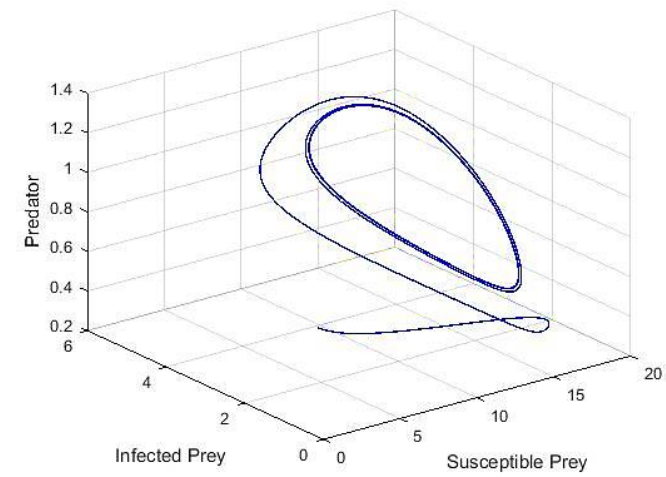

(a) $\sigma=0.21$

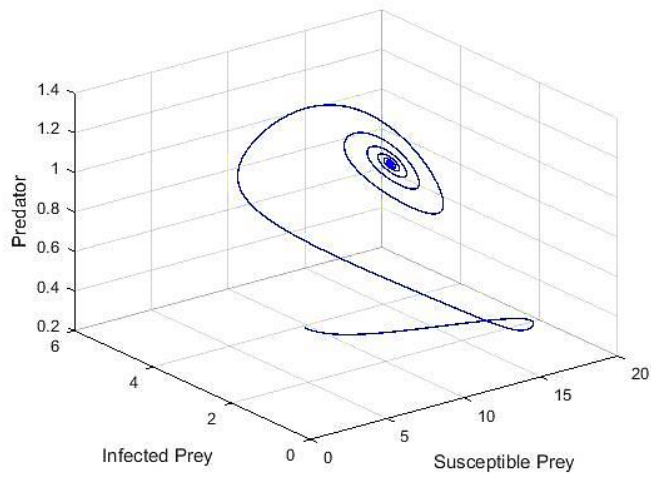

(b) $\sigma=0.3$

Figure 2. Phase portrait with different values of $\sigma$, the change of stability from unstable to stable 


\section{Adin Lazuardy Firdiansyah}

To see the effect of the harvesting rate in the system (1), we observe the dynamics behavior of the system (1) by using different values of $h$. Figure 3 shows the time plot of each population which means that the size of each population decrease for at a long time and all trajectories converge rapidly.
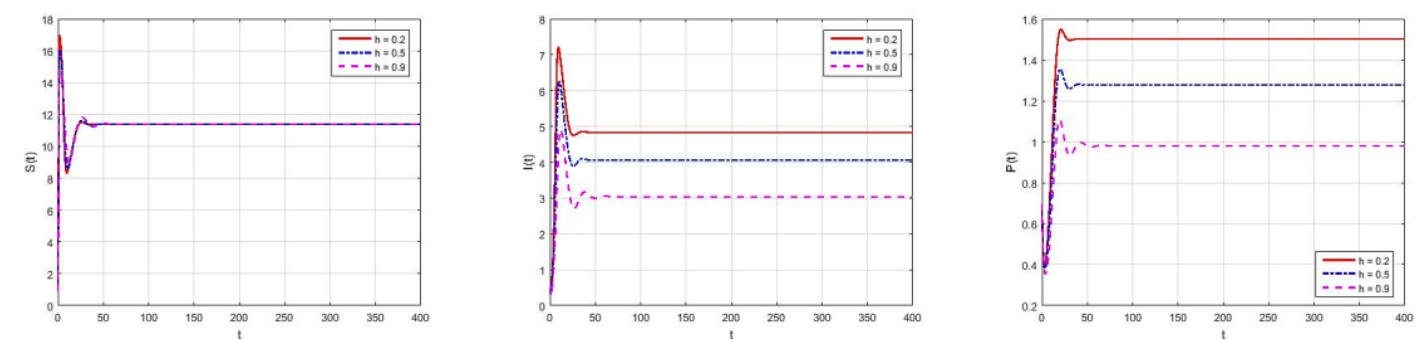

Figure 3. Influence of harvesting rate with different values of $h$

\section{Conclusion}

We have investigated an eco-epidemiology model of the modified Leslie-Gower model and Holling type III by incorporating refuging and harvesting on prey. In the model, we find at most six equilibrium points where three equilibrium points are locally asymptotically stable, i.e. axial equilibrium $E_{2}$, planar equilibrium $E_{4}$, and interior equilibrium $E^{*}$ and three equilibrium points are unstable. Here, we observe that infected prey refuge gives an important effect on stability. By taking $\sigma$ as the bifurcation parameter, Hopf bifurcation occurs when $\sigma=\sigma_{c}=0.26$. Thus, the condition of theorem 5 is satisfied. Furthermore, if the value of $\sigma$ is greater than critical values $\sigma_{c}=0.26$, then the phase portrait is stable. Meanwhile, if the value of $\sigma$ is less than critical values $\sigma_{c}=$ 0.26 , then the phase portrait is unstable. We also observe that by increasing the harvesting rate, the stability of all populations occurs faster. For future work, we can observe the global stability of the equilibrium point and influence of prey refuge.

\section{References}

[1] P. H. Leslie and J. C. Gower, "The Properties of a Stochastic Model for the Predator-Prey Type of Interaction Between Two Species," Biometrika, vol. 47, no. 3\&4, pp. 219-234, 1960, doi: 10.2307/2333294.

[2] Y. Cai, C. Zhao, W. Wang, and J. Wang, "Dynamics of a Leslie-Gower predatorprey model with additive Allee effect," Appl. Math. Model., vol. 39, no. 7, pp. 20922106, 2015, doi: 10.1016/j.apm.2014.09.038.

[3] H. S. Panigoro, E. Rahmi, N. Achmad, and S. L. Mahmud, "The Influence of Additive Allee Effect and Periodic Harvesting to the Dynamics of Leslie-Gower Predator-Prey Model," Jambura J. Math., vol. 2, no. 2, pp. 87-96, 2020, doi: 10.34312/jjom.v2i2.4566.

[4] M. Onana, B. Mewoli, and J. J. Tewa, "Hopf bifurcation analysis in a delayed Leslie-Gower predator-prey model incorporating additional food for predators, refuge and threshold harvesting of preys," Nonlinear Dyn., vol. 100, no. 3, pp. 3007-3028, 2020, doi: 10.1007/s11071-020-05659-7.

[5] S. Sharma and G. P. Samanta, "A Leslie-Gower predator-prey model with disease in prey incorporating a prey refuge," Chaos, Solitons and Fractals, vol. 70, no. 1, pp. 
Effect of Prey Refuge and Harvesting on Dynamics of Eco-epidemiology Model ...

69-84, 2015, doi: 10.1016/j.chaos.2014.11.010.

[6] J. J. Zhao, M. Zhao, and H. Yu, "Effect of prey refuge on the spatiotemporal dynamics of a modified leslie-gower predator-prey system with holling type III schemes," Entropy, vol. 15, no. 6, pp. 2431-2447, 2013, doi: 10.3390/e15062431.

[7] A. S. Purnomo, I. Darti, and A. Suryanto, "Dynamics of eco-epidemiological model with harvesting," AIP Conf. Proc., vol. 1913, 2017, doi: 10.1063/1.5016652.

[8] X. Zhou, J. Cui, X. Shi, and X. Song, "A modified Leslie-Gower predator-prey model with prey infection," J. Appl. Math. Comput., vol. 33, no. 1-2, pp. 471-487, 2010, doi: 10.1007/s12190-009-0298-6.

[9] R. Bhattacharyya and B. Mukhopadhyay, “On an eco-epidemiological model with prey harvesting and predator switching: Local and global perspectives," Nonlinear Anal. Real World Appl., vol. 11, no. 5, pp. 3824-3833, 2010, doi: 10.1016/j.nonrwa.2010.02.012.

[10] N. Apreutesei and G. Dimitriu, “On a prey-predator reactiondiffusion system with Holling type III functional response," J. Comput. Appl. Math., vol. 235, no. 2, pp. 366-379, 2010, doi: 10.1016/j.cam.2010.05.040.

[11] A. A. Shaikh, H. Das, and N. Ali, "Study of a predator-prey model with modified Leslie-Gower and Holling type III schemes," Model. Earth Syst. Environ., vol. 4, no. 2, pp. 527-533, 2018, doi: 10.1007/s40808-018-0441-1.

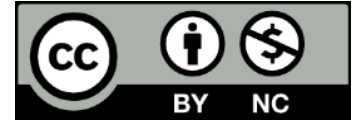

This article is an open access article distributed under the terms and conditions of the Creative Commons Attribution-NonCommercial 4.0 International License. Editorial of JoM: Department of Mathematics, Universitas Negeri Gorontalo, Jln. Prof. Dr. Ing. B.J. Habibie, Moutong, Tilongkabila, Kabupaten Bone Bolango, Provinsi Gorontalo 96119, Indonesia. 